\title{
Expression of human G3BP1 in E. coli
}

\author{
Ziwei $\mathrm{YANG}^{1 *}$ \\ ${ }^{1}$ School of Pharmaceutical Science and Technology, Tianjin University, Tianjin, 300072, China
}

\begin{abstract}
G3BP1 (Ras-GTPase-activating protein SH3 domain-binding protein) is responsible for normal RNA stress granule (SG) assembly and overexpressed in many cancer cells. Deletion of G3BP1 decreases the number and size of SGs. SGs are complex of RNA and proteins that stall translation of protein in response to stress. Given the function of G3BP1 in stress granule assembly and tumor suppression, it is believed that G3BP1 regulates cell growth and proliferation as well. Here, I constructed the recombinant protein expression vector and systemically optimized condition for the expression of human G3BP1 protein in E. coli. This research should be useful for investigating further functional analysis and atomic structure of G3BP1.
\end{abstract}

\section{Introduction}

Ras-GTPase-activating protein (GAP)-binding protein 1 ( G3BP1) was originally identified as the protein binding to the Ras-GTPase activating protein. It consists of four main domains: a nuclear transport factor 2 (NTF2) like domain, acidic and proline-rich regions (PXXP motif), an RNA recognition motif (RRM) and arginine and glycine rich boxes (Figure 1). NTF2 is necessary for G3BP1 in the nucleus during serum stimulation. It can also modulate conformation of G3BP1 through dimerization. PXXP motif is associated with the protein interaction. RRM motif can bind to and regulate the stability of cell development relevant RNA. RGG box is responsible for RNA binding and the interaction of G3BP1 with 40S ribosomal subunit to mediate stress granule assembly ${ }^{[1]}$. Stress granules are the cytosolic aggregations composed of RNA and protein. It is proposed to protect RNAs from harmful stress stimuli ${ }^{2}$, 3].

G3BP1 is necessary for stress granule assembly. Under stress condition (heat, cold, oxidative stress), Ser149 of G3BP1 is phosphorylated and G3BP1 undergoes open

conformation. The phosphorylation promotes dimerization and exposes RRM and RGG domains for interaction with RNA to form SGs. When not phosphorylated, it adopts a closed conformation to hide the RNA binding domain ${ }^{[4-6]}$. Many studies have demonstrated that hundreds of proteins and RNAs are the components of SGs, but have not revealed how these components contribute each other in what extent. Earlier studies discovered that G3BP1 is overexpressed in many cancer cells, such as lung cancer, colon cancer and breast cancer $^{[7]}$. It can directly bind to tumor suppressor $\mathrm{p} 53^{[8,9]}$. The depletion of G3BP1 can enhance the level of $\mathrm{p} 53^{[9]}$. G3BP1 has become an important biomarker of poor prognosis in multiple cancers, and a potential target for cancer therapy ${ }^{[10]}$. A novel research showed that G3BP1 is critical for DNA sensing and for efficient activation of cyclic GMP-AMP synthase (cGAS) through promoting the formation of large cGAS complexes ${ }^{[11]}$. G3BP1 can form a big complex with cGAS to trigger DNA binding. To biochemically characterize the domain structure, and the interaction with other binding partners, a convenient method to obtain purified G3BP1 is necessary. Such purified protein should also facilitate understanding of structure-function relationship and formation of SGs network.

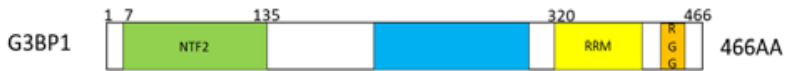

Fig. 1. Schematic structure of G3BP1. Nuclear transport factor 2-like (NTF2-like) domain, proline rich (PXXP) motif, RNA recognition motif (RRM), and loosely conserved arginine-glycine-glycine rich box ( $\mathrm{RGG}$, a.k.a. intrinsically disordered region 3, IDR3).

\section{Methods}

\subsection{Plasmid construction}

Human G3BP1 cDNA (UniProt Accession ID Q13283) were amplified by PCR using 5' primer ATGGGATCCATGGTGATGGAGAAGCCTAGTCCC, 3' primer GCTTCGAATTCTCACTGCCGTGGCGCAAG.

HEK293 cDNA library was used as templates and the PCR product was ligated into pGEX4T-1 vectors at BamH I/EcoR I sites, respectively. The target gene of G3BP1 was amplified by PCR, pGEX-4T1-G3BP1 was used as the template, after purification, the target gene was ligated onto pGEX-4T1-HTb vector at BamH I/EcoR I sites. 


\subsection{Optimization of conditions for G3BP1 expression in E. coli}

For the bacteria expression and purification of GST-G3BP1, the indicated plasmid pGEX-4T1-G3BP1 was transformed into E. coli BL21 following the transformation method and the bacteria was shaken in $500 \mathrm{ml} \mathrm{LB}$ medium. The protein expression was induced using $1 \mathrm{mM}$ IPTG when the OD reached 0.6. After $2 \mathrm{hr}$ induction, the bacteria was collected with centrifudgation for $10 \mathrm{~min}$ at $6000 \mathrm{rpm}$. The bacteria pellet was rinsed with $15 \mathrm{ml}$ of cold PBS followed by lysing with lysis buffer (PBS, pH 7.4, 5 mM EGTA, 0.1\% Tween 20 and $100 \mathrm{mM}$ PMSF), frozen in liquid nitrogen and stored at $-80{ }^{\circ} \mathrm{C}$. The frozen cells were thawed in $37{ }^{\circ} \mathrm{C}$ water bath and picked up before complete thawing. The bacteria suspension was sonicated at the condition of $30 \mathrm{sec}$ on/ $30 \mathrm{sec}$ off, totally $5 \mathrm{~min}$ until the solution is clear. To check expression, $20 \mu \mathrm{l}$ sample was taken and mixed with $10 \mu \mathrm{l} 3 \times$ SDS sample buffer. After centrifugation at $10000 \mathrm{rpm}, 20 \mathrm{~min}$ at $4{ }^{\circ} \mathrm{C}, 20 \mu \mathrm{l}$ of the supernatant was taken and other supernatant was incubated with $2 \mathrm{ml}$ of glutathione-sepharose beads at $4{ }^{\circ} \mathrm{C}$ for $1 \mathrm{~h}$ with gentle rocking. The beads were washed with buffer $\mathrm{P}$ (PBS, $\mathrm{pH}$ 7.4; $100 \mathrm{mM} \mathrm{NaCl} ; 1 \mathrm{mM} \beta-\mathrm{Me}$ and $0.1 \%$ Tween 20 ) and the glutathione resin was transferred to the column, washed with buffer $\mathrm{P}$ followed by $5 \mathrm{ml}$ of $50 \mathrm{mM}$ Tris-HCl, $\mathrm{pH}$ 8.0. The GST fusion protein was eluted with $10 \mathrm{mM}$ glutathione in $50 \mathrm{mM}$ Tris- $\mathrm{HCl}, \mathrm{pH} 8.0$. Four fractions were collected and the protein expression was checked by SDS-PAGE. The fractions were collected and desalted using PD-10 desalting columns packed with Sephadex G-25 resin (GE). For the purification of GST-His-G3BP1, the expression vector was transformed into E. coli BL21 (DE3) competent cells, cultured at $37{ }^{\circ} \mathrm{C}$ in $20 \mathrm{~mL} \mathrm{LB}$ medium until the OD600 value reached to $0.35 \sim 0.5$. The expression of the fusion protein was induced with $0.4 \mathrm{mM}$ IPTG for 2 hours at $37^{\circ} \mathrm{C}$.

\subsection{Protein electrophoresis and immunoblotting}

Samples were loaded onto 9\% SDS-PAGE gel. Separated proteins were transferred to nitrocellulose membrane and blocked with blocking buffer $(5 \%$ non-fat milk powder in TBST (20 mM Tris-HCl, pH 7.4, $110 \mathrm{mM} \mathrm{NaCl}, 5 \mathrm{mM}$ $\mathrm{MgCl} 2,0.1 \%$ Tween 20). Primary antibodies were prepared in the blocking solution and membranes were incubated overnight at $4{ }^{\circ} \mathrm{C}$. The membrane was washed in TBST and incubated with HRP-conjugated secondary antibodies in the blocking buffer for $1 \mathrm{~h}$ at room temperature. The membrane was washed and developed with the HRP substrate (WesternBright ECL, Advansta).

\section{Results}

We successfully amplified human G3BP1 from HEK 293A cDNA library and inserted it into the vector. Sequence alignment indicated the correction of target gene. In order to biochemically characterize G3BP1, large quantities of target protein is required. The temperature was set $18{ }^{\circ} \mathrm{C}$ or $37{ }^{\circ} \mathrm{C}$ and concentration of IPTG was set at $0.4 \mathrm{mM}$ and $1 \mathrm{mM}$, respectively. The optimized expression condition was $1 \mathrm{mM}$ IPTG and induction for $2 \mathrm{hr}$ at $37{ }^{\circ} \mathrm{C}$. In this condition, high amount of soluble target protein was obtained (Figure 2). From $500 \mathrm{ml}$ of the bacteria culture, $\sim 5 \mathrm{mg}$ of purified GST-G3BP1 was obtained.

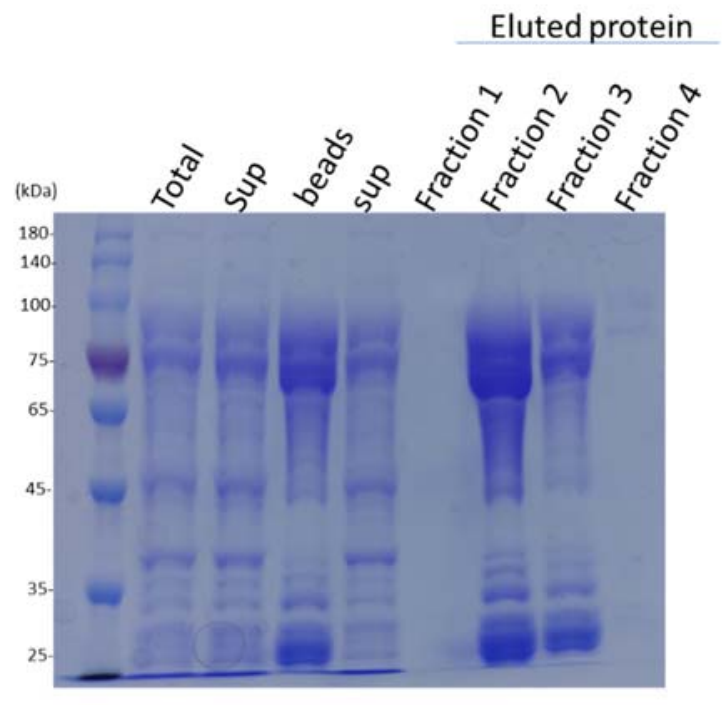

Fig. 2. Protein expression of pGEX-4T-G3BP1 in E. coli (BL21) cells was detected by CBB (coomasie brilliant blue) staining. Protein expressing in bacteria were induced at $37^{\circ} \mathrm{C}$ with $1 \mathrm{mM}$ IPTG for $2 \mathrm{~h}$. The total lysate and supernatant were separated by $9 \%$ SDS-PAGE gel. The supernatant was collected and immobilized on glutathione-sepharose beads. Unbound protein was washed away and bound protein was eluted by $10 \mathrm{mM}$ glutathione in $50 \mathrm{mM}$ Tris- $\mathrm{HCl}, \mathrm{pH}$ 8.0. Four fractions were collected. The resin, washed fraction and four eluted fractions was added in SDS sample buffer and prepared for SDS-PAGE. Protein expression of GST-G3BP1 showed the target protein was mainly in the supernatant, and G3BP1 fused to GST can immobilize on the glutathione beads. GST fusion G3BP1 was mainly stay in the second and third fraction. Arrows indicated positions of the target protein. total: total lysate; sup: supernatant; beads: glutathione-sepharose resin.

The purified GST-G3BP1 protein was confirmed by western blot using mouse monoclonal G3BP1 antibody. Briefly, 10 ng protein solution were mixed with $3 \times$ SDS sample buffer and prepared for SDS-PAGE. The separated protein was transferred to the NC membrane at the condition of $18 \mathrm{~V}$ for $1 \mathrm{hr}$ using semi-dry transfer method. After antibody incubation and chemilluminescence, $75 \mathrm{kDa}$ was detected as expected (Figure 3). 


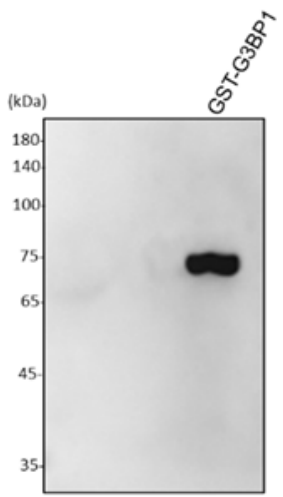

Fig. 3. Protein expression of GST-G3BP1 in BL 21 (DE3) competent cell. Protein expression in bacteria was induced at $37^{\circ} \mathrm{C}$ with $1 \mathrm{mM}$ IPTG for $2 \mathrm{~h}$. Protein expression level of GST-G3BP1 was confirmed by western blotting using anti-G3BP1 antibody.

I also constructed pGEX-4T-HTb-G3BP1, human G3BP1 fused to GST-His tag which can be cleaved off by TEV protease. The condition was optimized as described below. For protein induction, different temperatures were tested at $18{ }^{\circ} \mathrm{C}, 30^{\circ} \mathrm{C}$ and $37^{\circ} \mathrm{C}$. The concentration of IPTG was also varied at $0.1 \mathrm{mM}, 0.4$ $\mathrm{mM}$ and $1 \mathrm{mM}$. In total, nine different conditions were tested as shown in Figure 4. Under the condition of using $0.4 \mathrm{mM}$ IPTG to induce for $2 \mathrm{hr}, 4 \mathrm{mg}$ of protein was obtained with $500 \mathrm{ml}$ of bacteria medium. In this bacteria expression system, GST tag can be cleaved by thrombin and His tag fusion protein can be used for biological function assay as well. GST can enhance solubility of target protein, but it may affect protein function and downstream experiments. Therefore, it can be immobilized on the glutathione beads and CBB staining indicated the right position corresponding to the predicted molecular weight. It can be used for pull down assay and immune precipitation with the protein immobilized on the beads, which will help validate protein interaction in vitro. There are two reasons for GST application in prokaryotic expression of human G3BP1. One is that it is a highly soluble protein, which can be used to increase the solubility of target protein; The other is that which may interfere functional assay in vitro. Since the high-resolution structure of G3BP1 is not known and it requires large amount of purified protein for crystallization, the present work should be used to prepare protein reagent for the crystallization.

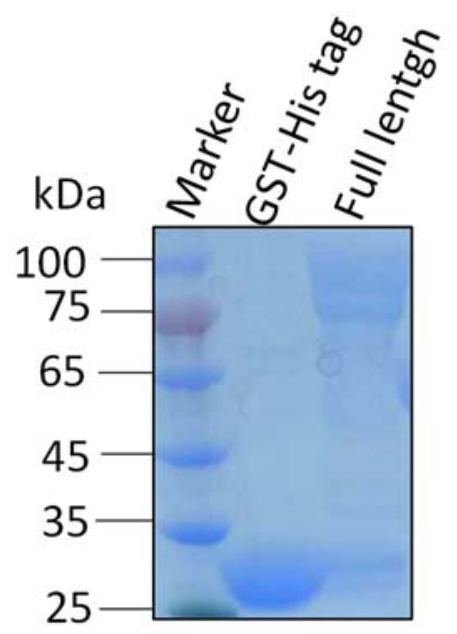

Fig. 4. Protein expression of pGEX-4T-HTb-G3BP1 in E. coli (BL21) cells was detected by CBB (coomassie brilliant blue) staining. Protein expressing in bacteria were induced at $37^{\circ} \mathrm{C}$ with $0.4 \mathrm{mM}$ IPTG for $2 \mathrm{~h}$. GST-His, GST-His-G3BP1 (full length) were loaded onto $9 \%$ SDS-PAGE and the gel was stained with Coomassie Brilliant Blue.

\section{Conclusion}

Given the function of G3BP1 in cancer progression and stress granule formation, it is important to prepare purified protein for functional analysis in vitro. In this research, I have established a method to express and purify human G3BP1 with high yield. Moreover, I have successfully expressed full-length and fragments of G3BP1 as a GST-His protein. The fusion protein can be used for investigating downstream experiments such as con-sedimentation assay to discover a potential new binding partner. It can be also used for competition assay between RNA and proteins that potentially bind to RNA-binding sites of G3BP1. The purified protein can also be used for high-resolution structural analysis. The atomic structure of G3BP1 should help for better understanding of the interaction of G3BP1 with other protein or RNA. Such structural information should also help to solve a problem regarding how it is modulated. Since ample evidence suggests that G3BP1 is a novel target for cancer therapy, large amount of purified G3BP1 can be used for drug screening as well. In conclusion, we established the purification method for recombinant GST fused proteins of full-length and fragments of G3BP1. Therefore, this protocol is expected to promote the biochemical research of G3BP1-related physiology and pathology.

\section{References}

1. U. Alam, D. Kennedy, Biochim Biophys. Acta Mol Cell Res, 1866: 360-370, (2019).

2. S P. Somasekharan, A. El-Naggar, G Leprivier. J Cell Biol, 208: 913-929, (2015).

3. A. Aulas, G. Caron, C G. Gkogkas. J Cell Biol, 209: 
73-84, (2015).

4. P. Yang, C. Mathieu, R M. Kolaitis. Cell, 181: 325-345, (2020).

5. J. Guillen-Boixet, A. Kopach, A S. Holehouse. Cell, 181: 346-361, (2020).

6. H. Sidibe, A. Dubinski, C. Vandevelde. J Neurochem, (2021).

7. K. Taniuchi, I. Nishimori, M A. Hollingsworth. Mol Cancer Res, 9: 856-866, (2011).

8. M M. Kim, D. Wiederschain, D. Kennedy. Oncogene, 26(29): 4209-4215, (2007).

9. Mao C, Wang X, Liu Y T. Cancer Res, 78(13): 3484-3496, (2018).

10. N. Oi, J. Yuan, M. Malakhova. Oncogene, 34(20): 2660-2671, (2015).

11. Liu Z S, Cai H, Xue W. Nat Immunol, 20(1): 18-28, (2019). 